\title{
Dynamics of the Tip Vortices in the Wake Behind a Circular Cylinder of Finite Length
}

\author{
Václav Uruba ${ }^{1,2, *}$, Pavel Procházka ${ }^{1}$ \\ ${ }^{1}$ Department of Fluid Dynamics, Institute of Thermomechanics of the Czech Academy of Sciences, \\ Dolejškova 5, 182 00, Czech Republic \\ ${ }^{2}$ Department of Power System Engineering, Faculty of Mechanical Engineering, University of West \\ Bohemia, Universitní 8, 30614 Plzeň, Czech Republic
}

\begin{abstract}
The dynamics of the tip vortices in the wake behind a wallmounted finite-length circular cylinder of the aspect ratio 2 was studied experimentally using time resolved stereo PIV technique. The cylinder was mounted normal to a ground plane and it was subjected to a cross-flow with thin boundary layer developed on the wall, the Reynolds number based on inflow velocity and cylinder diameter was 9.7 thousands. The dynamics of tip vortices were analysed using the POD method applied to the plane perpendicular to the flow close to the cylinder. Besides the decaying power spectrum, slower that the Kolmogorov-type one, the two distinct frequencies were detected on Strouhal numbers 0.09 and 0.15 . These frequencies could be linked to the vortical structures dynamics in the wake. The frequency $\mathrm{Sh}=0.15$ corresponds to predominantly spanwise vortices dynamics with anti-symmetrical patterns with respect to the cylinder axis, while the frequency $\mathrm{Sh}=0.09$ corresponds to mainly streamwise vortical structures dynamics with symmetrical patterns respectively. Thus, the von Kármán vortex street was detected on Strouhal frequency 0.15 .
\end{abstract}

\section{Introduction}

Infinitely long circular cylinder in cross-flow is one of the canonical cases of the fluid dynamics. This case is connected with periodical separation of vortical structures from the sides of a bluff-body, so called von Kármán-Bénard vortex-street. Many experimental studies and mathematical simulations could be find in literature (see e.g. $[1,3,4,8,10,17]$ ). However, every real cylinder is of a limited length, its effect could be quantified by so called aspect ratio defined as the follows: $\mathrm{AR}=L / D$, where $L$ stands for the cylinder length and $D$ for its diameter. There are numerous practical applications of the finite-length cylinder, obstacles in flow parts in turbo-machines or typical architectonic structures including buildings to give a few typical examples.

The wake dynamical behaviour of an infinite length cylinder is characterized by a pseudo-periodical phenomenon, i.e. von Kármán-Bénard vortex-street with its fundamental frequency. The dimensionless frequency is called Strouhal number and it is defined as

\footnotetext{
*Corresponding author: uruba@it.cas.cz
} 
follows: $\mathrm{St}=(f . D) / U$, where $f$ is the frequency, $D$ is the cylinder diameter and $U$ is the incoming velocity. Typical value of the Strouhal number corresponding to the fundamental frequency is about $\mathrm{St}=0.2$ in the broad range of Reynolds numbers, higher than 200 (see e.g. $[1,9])$.

The presented study brings some experimental results of wake dynamics behind a short cylinder. The finite-length circular cylinder of the subcritical aspect ratio $A R=2$ attached on a wall was studied experimentally using stereo PIV technique. The cylinder was mounted normal to a ground plane and it was subjected to a cross-flow with thin boundary layer developed on the wall. The Reynolds number based on inflow air velocity and cylinder diameter was 9.7 thousands. The dynamics of the wake is studied in the plane perpendicular to the flow. Basic frequency and respective flow dynamics are to be studied.

Unlike infinitely long circular cylinder in cross-flow the finite length cylinder is not studied thoroughly in the available literature, see e.g. $[12,13,14,15,16]$. In $[12,13,16]$ it is demonstrated, that the flow behind the cylinder of the aspect ratio higher than 5 is similar to that of the infinite cylinder with dominating Kármán-Bénárd vortex street, however deformed in a 3D manner, dominated by strong end-effects. The shorter cylinders are characterized by qualitatively different flow structure. In paper [15] the critical value of the AR is defined, bellow which the vortex shedding changes from the anti-symmetrical von Kármán type to the symmetric arch-type. The critical value of the aspect ratio $\mathrm{AR}_{\text {crit }}$ is reported to be between 2 and 6 (see e.g. [15]). The subcritical case is characterized by parallel vortex shedding in the wake. Then both the tip and base vortices resulted from the projection of the three-dimensional arch-type flow structure in the cross-section plane. The horse-shoe vortex originates in subsequence of interaction between the wall-boundary layer and the cylinder. Trailing (base) vortex is the result of the strong down-wash effect.

In the presented study only tip vortices and von Kármán type dynamics are to be studied in the near wake.

\section{Experimental Setup and Instrumentation}

The experimental setup and the experimental method used will be specified now.

\subsection{Experimental Setup}

The cylinder of diameter $15 \mathrm{~mm}$ is placed perpendicularly to the flow in the test-section inlet of the blown-down facility. The cross-section of the closed test-section is $250 \times 250 \mathrm{~mm}^{2}$ in the position of the cylinder. The velocity on the test-section inlet was $U_{\mathrm{e}}=5 \mathrm{~m} / \mathrm{s}$ corresponding to the Reynolds number about 9630 .

The situation is depicted schematically in Fig. 1, there is the cylinder (in green) mounted on the bottom wall (in violet), and plane of measurement (in grey). Cartesian coordinate system $\left(x_{\mathrm{r}}, y_{\mathrm{r}}, z_{\mathrm{r}}\right)$ with origin located on the cylinder axis, the coordinates are expressed in multiples of the cylinder diameter $D$ and thus dimensionless. The flow is in the $x_{\mathrm{r}}$ axis direction.

The plane of measurement was located in the position $x_{\mathrm{r}}=1.17$. Blockage of the test section cross-section was about $6 \%$, the boundary layer thickness in the cylinder position was about $2 \mathrm{~mm}$, that is $0.07 \mathrm{D}$. 


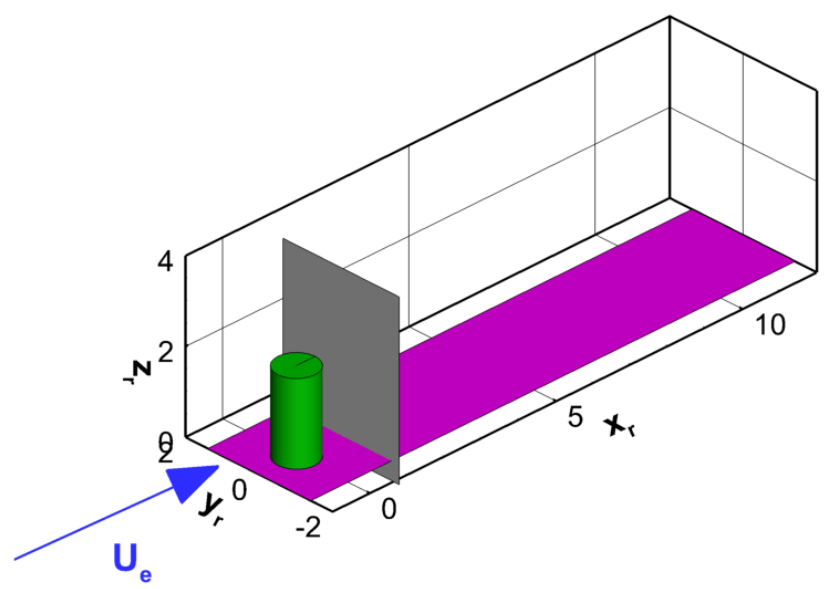

Fig. 1. Schema of experimental setup.

\subsection{Instrumentation}

The velocity vector fields were measured using Particle Image Velocimetry (PIV) method in stereo version.

The measurement apparatus consists of laser and 2 CMOS cameras by Dantec Company. The laser is New Wave Pegasus, Nd:YLF double head with wavelength of $527 \mathrm{~nm}$, with maximal frequency $10 \mathrm{kHz}$ and shot energy of $10 \mathrm{~mJ}$ (for $1 \mathrm{kHz}$ ), thus the corresponding power is $10 \mathrm{~W}$ per one head. The 2 cameras VEO 410 with resolution of $1280 \times 800$ pixels are able to acquire double snaps with frequency up to $2500 \mathrm{~Hz}$ (full resolution) and they use internal memory $16 \mathrm{~GB}$ each. The Scheinpflug mounting were used for the cameras lenses to get the focus planes identical with the laser-sheet plane. The stereo-PIV configuration is a standard one described e.g. in [2]. The data were acquired and post-processed using the Dynamic Studio and Tecplot software.

The PIV measurement was performed using acquisition frequency $2 \mathrm{kHz}, 4000$ doublesnapshots representing $2 \mathrm{~s}$ in real time were acquired. The Safex generator of particles in the form of oil droplets was used, the mean diameter of the particles was $1 \mu \mathrm{m}$.

The stereo PIV method provides all 3 velocity vector components, which were evaluated in the plane of measurement. More detailed description of the experimental setup and procedures could be found in [6].

\section{Results}

The results are to be presented in dimensionless form. The distances are expressed in multiples of the cylinder diameter $D$ and the velocities in multiples of the incoming velocity $U_{\text {e. }}$ All other quantities (as vorticity or TKE) are non-dimensioned with help of the $D$ and $U_{\mathrm{e}}$ as well.

The plane of measurement is shown in the positive $x_{\mathrm{r}}$ axis direction.

\subsection{The Mean Flow-Field}

The flow-field is to be shown in terms of time-mean quantities first. 
In Fig. 2 the time mean velocity vector field is shown. For the sake of clarity, the corresponding vector-lines in black are added in an arbitrary manner.

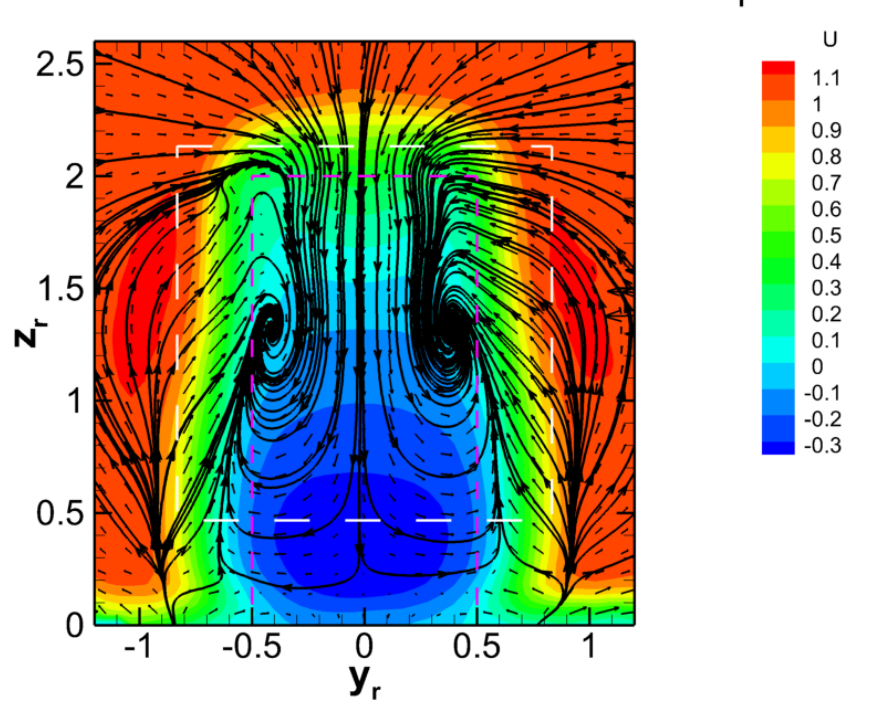

Fig. 2. Mean velocity distribution in the plane of measurement.

In Fig. 2 the contour of the cylinder is shown by violet dashed line. Low velocity region (with back-flow) in the wake is depicted in blue, while outside the wake the velocity approaches the incoming velocity value $U_{\mathrm{e}}$, with even overshoot on sides. The down-wash effect close to the cylinder axis is evident as well as the pair of contra-rotating tip vortices in positions $y_{\mathrm{r}}=+-0.4$ and $z_{\mathrm{r}}=1.35$.

For the dynamical analysis only part of the flow-field was chosen bounded by white dashed rectangle in Fig. $2, y_{r} \in(-0.833 ; 0.833)$ and $z_{r} \in(0.473 ; 2.133)$. Hereinafter we will call it the Area of Interest (AoI).

The mean flow-field in this area is to be shown in details. In Fig. 3 again mean velocity distribution is depicted on the left, vectors represent the in-plane velocity components, while colour quantifies the out-of-plane velocity component. The white line delimits the back-flow region. In Fig. 3 on the right, the vector-lines from in-plane velocity components are shown together with the out-of-plane mean vorticity component (red positive, blue negative). The tip vortices are visualised in this way. 

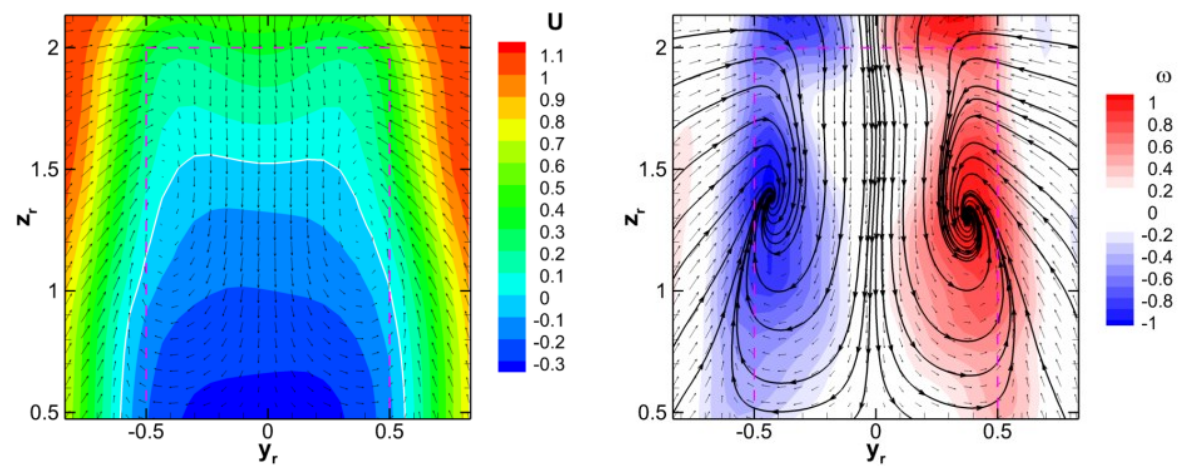

Fig. 3. Mean velocity and mean vorticity distributions in the AoI.

To demonstrate the velocity field dynamics, the Turbulence Kinetic Energy (TKE) was evaluated and its distribution is shown in Fig. 4, again in dimensionless form.

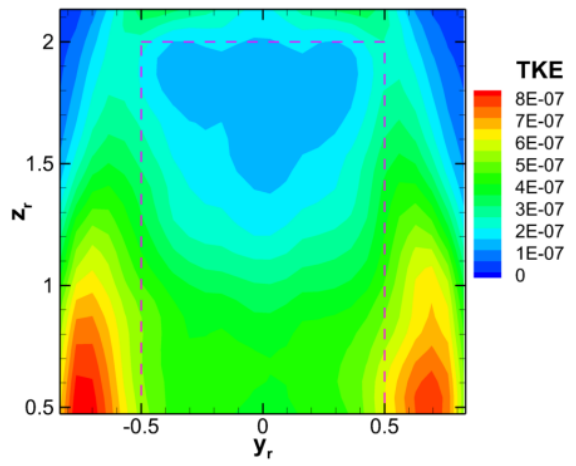

Fig. 4. TKE distribution in the AoI.

The blue regions are calm, while the red colour indicates high fluctuating activity. The two high activity regions are located close to the cylinder contour in the bottom AoI part.

\subsection{The Proper Orthogonal Decomposition}

The Proper Orthogonal Decomposition (hereinafter POD) is considered as a basic tool to study dynamics of extended dynamical systems, e.g. turbulent flows, see e.g. $[2,5,11]$. The POD provides an unbiased technique for identifying deterministic features from a random, fine grained turbulent flow. The method extracts the best correlated structures in a statistical sense. A set of structures is identified with the orthogonal eigenfunctions of the decomposition theorem of probability theory.

The goal of the decomposition methods in general is to look for a specific base with a distinct physical meaning. In the case of the POD that means an orthonormal basis corresponding to decorrelated (i.e. orthogonal) modes maximizing the spatio-temporal data variance. In the case of application on velocity field the maximized quantity physical interpretation is a total turbulent kinetic energy, i.e. sum of all TKE within the analyses region.

In this paper we will consider the POD in its extended version, which uses decomposition in both spatial and temporal domains. This version is also called Bi- 
Orthogonal Decomposition, see e.g. [5]. Each mode is to be represented by its topological (Topos) and temporal (Chronos) parts.

The result of the POD analysis is to be presented. The set of 4000 snapshots was decomposed using a standard POD procedure implemented in the DynamicStudio software resulting in the 4000 POD modes. In Fig. 5 the kinetic energy distribution among the POD modes is shown. The first quantity the Energy Fraction (EF) express the percentage shear of a given POD mode on the total kinetic energy, the Accumulated Energy (AE) expresses the sum energy of all lower order POD modes and Residual Energy (RE) is an unexplained part of the total kinetic energy by a given set of modes.

Please note that the Total Kinetic Energy is the sum of the Turbulence Kinetic Energy within the AoI.

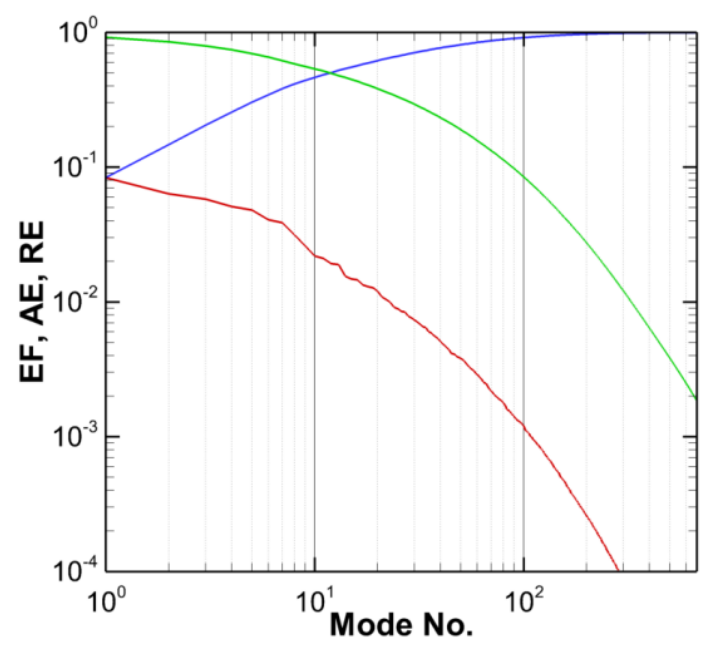

Fig. 5. Kinetic energy distribution among the POD modes.

The first 10 POD modes cover only about $46 \%$ of the total kinetic energy. The first 4 modes, which are to be shown below in details, contain $8.4,6.3,5.8$ and $5.1 \%$ of the total kinetic energy respectively.

The temporal parts of the POD modes are to be analysed first, as they play the crucial role in the dynamics. The power-spectra were evaluated.

In Fig. 6 the power-spectra for the modes 1, 2, 3 and 4 Chronoses are shown in log-log representation. The frequencies are shown in dimensionless form as Strouhal numbers Sh. The pink full line represents the Kolmogorov law characterized by the slope $-5 / 3$ and the dashed line is the most representative slope of the turbulent part of the analysed POD modes, characterized by the slope about $-7 / 6$.

The spectra in Fig. 6 show some peaks and slopes. For the small frequencies up to Sh about 0.08 the spectra are more or less constant. The high frequencies, Sh 0.2 and higher, the spectra obey the decay law, however the slope is about $-7 / 6$, rather far from Kolmogorov law, not so steep. This could be effect of not well developed turbulence very close behind the cylinder, which is dominated by quasi-regular vortical structures, which are fare from to be isotropic.

The dashed line marks the $\mathrm{Sh}=0.15$, modes 1 and 2 show maxima on this frequency. The dotted line is on $\mathrm{Sh}=0.09$, the modes 3 and 4 have their maxima here.

The spectra of higher order POD modes contain no distinct peaks on any frequency, they are basically dense with slower or steeper decay, maximum slope again $-7 / 6$. 


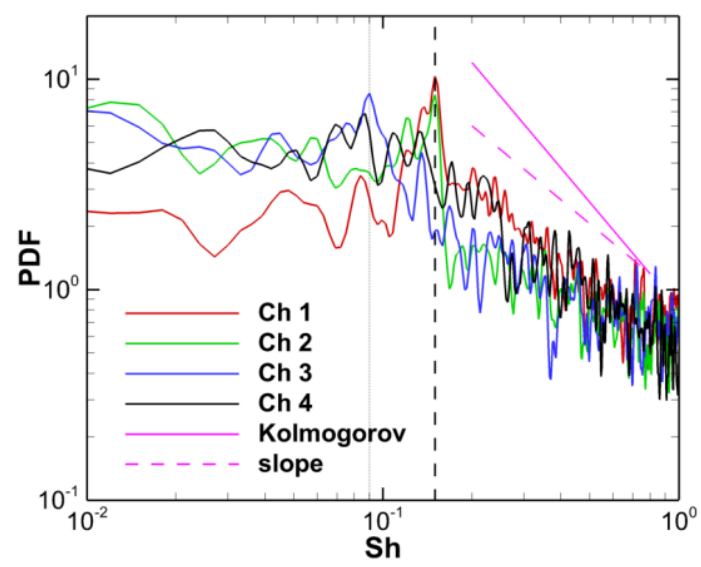

Fig. 6. Spectra of the Chronoses mode $1,2,3$ and 4 .

The corresponding Toposes are to be shown as well. The in-plane velocity component fluctuations are shown as vectors boosted by vector-lines. The colour represents the out-ofplane velocity fluctuation component, in blue negative, in red positive. Absolute values has no meaning, only patterns matters.

The first two POD modes characterized by the frequency peak on $\mathrm{Sh}=0.15$ are in Fig. 7. The fluctuating velocity vectors distributions are close to be anti-symmetrical with respect to the cylinder axis (dash-dotted).
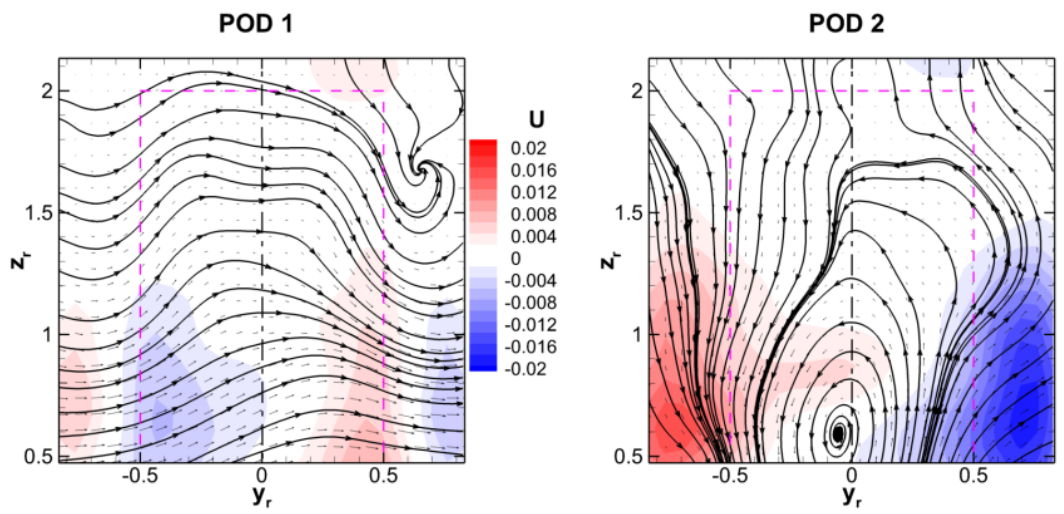

Fig. 7. Toposes of the POD modes 1 and 2.

Those structures could be related to alternative shedding from the cylinder sides. Especially the POD mode 1 represents well the vortex shedding of von Kármán type, as horizontal vector-lines are present. The POD mode 2 is dominated by a streamwise vortex with its centre on the axis, streamwise component of fluctuating velocity are positive and negative on sides. However, the anti-symmetrical character of the modes 1 and 2 indicates presence of spanwise oriented structures, parallel to the cylinder axis, appearing in alternative manner.

The POD modes 3 and 4 characterized by $\mathrm{Sh}=0.09$ are shown in Fig. 8 . The patterns are more or less symmetrical with respect to the cylinder axis. The mode 3 is dominated by a vortex on the right-hand-side, on the left the vortex is a little degenerated, but there is a 
sign of it. The mode 4 consists of 4 vortices in symmetrical configuration. The streamwise component distribution is close to symmetry for both modes. This could be connected with appearance of spanwise structures shed parallel.
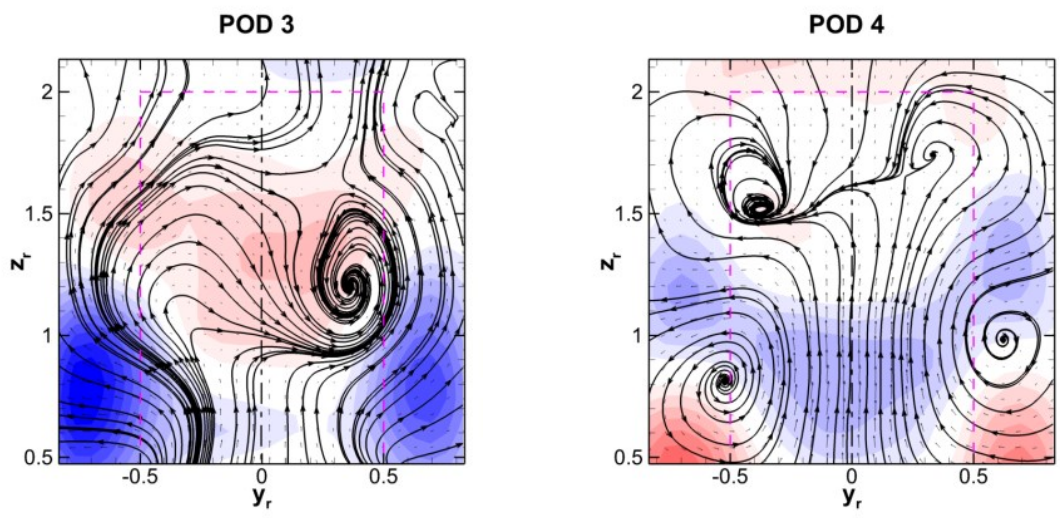

Fig. 8. Toposes of the POD modes 3 and 4.

The 3 and 4 POD modes are dominated by streamwise vortical structures in a pattern close to be symmetrical.

The higher order POD modes have no distinct peak in theirs Chronos spectra and the corresponding Toposes look to be not ordered in the sense of symmetry. The higher order, the more complex and chaotic the topology is. As an example the POD modes 30 and 100 are shown in Fig. 9.
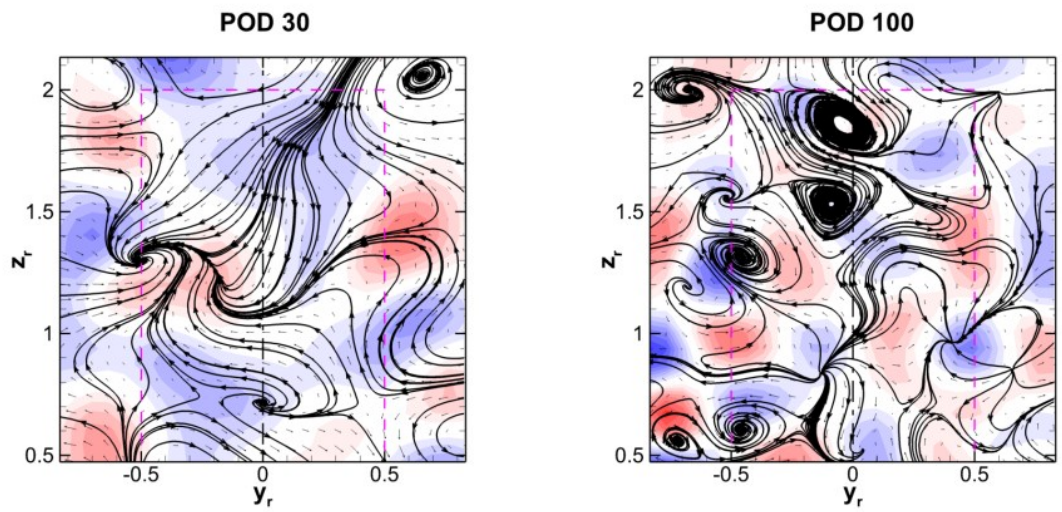

Fig. 9. Toposes of the POD modes 30 and 100.

The Toposes are dominated by randomly distributed streamwise vortices and spots of positive-negative streamwise velocity fluctuations.

In general, the tendency of the high order modes is towards white noise character of the Chronos spectrum and increasing complexity and randomness of the Topos structure.

The chaotic character of POD modes was studied in [6].

\section{Conclusions}

The dynamics of the tip vortices in the wake behind a wall-mounted finite-length circular cylinder was studied experimentally. 
The cylinder $15 \mathrm{~mm}$ in diameter was mounted normal to a ground plane and it was subjected to a cross-flow $5 \mathrm{~m} / \mathrm{s}$ with thin boundary layer developed on the wall. The Reynolds number based on inflow velocity and cylinder diameter was 9.7 thousands. The aspect ratio of the cylinder was 2 , having the length $30 \mathrm{~mm}$. Thus the aspect ratio was subcritical producing different wake structure than a long cylinder.

For experiments the time resolved stereo PIV technique was used for detecting full velocity vectors in a plane perpendicular to the inflow in the near cylinder wake. Only part of the wake containing tip vortices was studied. Besides the flow statistics, the POD method was applied to decompose the flow dynamics into coherent modes. Both temporal and spatial analysis have been performed.

The analysis of modes time evolution by Fourier spectra the 2 distinct frequencies and decay law were detected. The Strouhal numbers corresponding to the detected frequencies were numbers 0.09 and 0.15 . The decay law exponent of the power spectrum $-7 / 6$ was ascertained, considerably smaller than the Kolmogorov-type one.

The detected frequencies are linked to the vortical structures dynamics in the wake. The frequency $\mathrm{Sh}=0.15$ corresponds to predominantly spanwise vortices dynamics with antisymmetrical patterns with respect to the cylinder axis, those structures correspond to von Kármán type alternative vortex shedding phenomenon. The frequency $\mathrm{Sh}=0.09$ corresponds to mainly streamwise vortical structures dynamics with symmetrical geometry. Those dynamical structures are characterized by synchronous dynamical behaviour.

The authors acknowledge the financial support of the work by the Grant Agency of the Czech Republic, projects Nos. 17-01088S, 19-04695S and 19-02288J.

\section{References}

1. Roshko A.: On the development of turbulent wakes from vortex streets. NACA Rep. 1191, pp.801-825 (1955)

2. Procházka P., Uruba V.: Streamwise and spanwise vortical structure merging inside the wake of an inclined flat plate, Mechanics\&Industry, 20, Issue 7, (2019)

3. Uruba V.: On 3D instability of wake behind a cylinder, AIP Conference Proceedings, 1745, (2016), Art. no. 020062

4. Uruba, V., Procházka, P.: The Reynolds number effect on dynamics of the wake behind a circular cylinder. AIP Conference Proceedings, 2189, (2019), Article number 020023

5. Uruba V.: Decomposition methods in turbulent research, EPJ Web of Conferences, 25 01095 (2012)

6. Uruba, V.: Energy and Entropy in Turbulence Decompositions, ENTROPY, 21, 2, Article Number: 124, (2019), 18pp.

7. Uruba V., Pavlík D., Procházka P., Skála V., Kopecký V.: On 3D flow-structures behind an inclined plate, EPJ Web of Conferences, 143, Article number 02137 (2017)

8. Williamson, C.H.K.: Vortex Dynamics in the Cylinder Wake, Annu. Rev. Fluid. Mech. (1996) 28, pp 477-539

9. White, F.M.: Fluid Mechanics. McGraw Hill, (2015).

10. Uruba V., On 3D instability of wake behind a cylinder, AIP Conference Proceedings, Vol 1745, 30 June 2016, Art. no. 020062 
11. Uruba V., Near Wake Dynamics around a Vibrating Airfoil by Means of PIV and Oscillation Pattern Decomposition at Reynolds Number of 65 000, Journal of Fluids and Structures, 55, pp. 372-383 (2015)

12. Amor Ch., Pérez J., Schlatter P., Vinuesa R., Le Clainche S., Modeling the turbulent wake behind a wall-mounted square cylinder. Logic Journal of IGPL. 1-11. 10.1093/OUP. (2020)

13. Johnston C.R., Wilson D.J., A Vortex Pair Model for Plume Downwash into Stack Wakes, Atmospheric Environment, Vol. 31, No.1, pp. 13-30 (1997)

14. Krajnovic S., Flow around a tall finite cylinder explored by large eddy simulation, J. Fluid Mech., Vol. 676, 2011, pp. 294-317

15. Norberg C., An experimental investigation of the flow around a circular cylinder: influence of aspect ratio, J. Fluid Mech. (1994), vol. 258, pp. 287-316

16. Palau-Salvador G., Stoesser T., Frohlich J., Kappler M., \& Rodi W., 2010 Large eddy simulations and experiments of flow around finite-height cylinders. Flow Turbul. Combust. 84, pp239-275.

17. Procházka P. and Uruba V., Reynolds Number Effect on Velocity Field and on Coherent Structures behind a Cylinder, AIP Conf. Proc. 2118, 030037-1-030037-4; (2019) 